\title{
Evolution of an archaeal virus nucleocapsid protein from the CRISPR-associated Cas4 nuclease
}

\author{
Mart Krupovic $^{1 *}$, Virginija Cvirkaite-Krupovic ${ }^{1}$, David Prangishvili ${ }^{1}$ and Eugene V. Koonin ${ }^{2}$
}

\begin{abstract}
Many proteins of viruses infecting hyperthermophilic Crenarchaeota have no detectable homologs in current databases, hampering our understanding of viral evolution. We used sensitive database search methods and structural modeling to show that a nucleocapsid protein (TP1) of Thermoproteus tenax virus 1 (TTV1) is a derivative of the Cas4 nuclease, a component of the CRISPR-Cas adaptive immunity system that is encoded also by several archaeal viruses. In TTV1, the Cas4 gene was split into two, with the N-terminal portion becoming TP1, and lost some of the catalytic amino acid residues, apparently resulting in the inactivation of the nuclease. To our knowledge, this is the first described case of exaptation of an enzyme for a virus capsid protein function.
\end{abstract}

Reviewers: This article was reviewed by Vivek Anantharaman, Christine Orengo and Mircea Podar. For complete reviews, see the Reviewers' reports section.

Keywords: Virus evolution, Capsid proteins, Nucleocapsid, Virus origin, Archaea viruses

\section{Findings}

The ability to form virions is the key feature which distinguishes viruses from other types of mobile genetic elements, such as plasmids and transposons [1-7]. The origin of bona fide viruses thus appears to be intimately linked to and likely concomitant with the origin of the capsids. However, tracing the provenance of viral capsid proteins (CPs) proved to be particularly challenging because they typically do not display sequence or structural similarity to proteins from cellular life forms [1, 8]. Over the years, a number of structural folds have been discovered in viral CPs. Strikingly, morphologically similar viral capsids, in particular, icosahedral, spindle-shaped and filamentous ones, can be built from CPs which have unrelated folds [8-11]. Thus, in the course of evolution, viruses have found multiple solutions to the same problem [12]. Nevertheless, the process of de novo origin of viral CPs remains largely enigmatic. Here we show that one of the capsid proteins of a filamentous archaeal virus, Thermoproteus tenax virus 1 (TTV1), evolved relatively

\footnotetext{
* Correspondence: krupovic@pasteur.fr

'Department of Microbiology, Unité Biologie Moléculaire du Gène chez les Extrêmophiles, Institut Pasteur, Paris 75015, France

Full list of author information is available at the end of the article
}

recently through exaptation from a CRISPR-associated Cas4 nuclease.

TTV1 was the first virus to be isolated from a hyperthermophilic archaeon [13]. It infects the crenarchaeon Thermoproteus tenax which grows optimally at $86{ }^{\circ} \mathrm{C}$. The virions are flexible filaments $400 \times 40 \mathrm{~nm}$ in size and consist of a linear, $16 \mathrm{~kb}$ dsDNA genome (Fig. 1a), four capsid proteins (TP1-4) and a lipid-containing envelope [14]. Dissociation experiments have shown that basic proteins TP1 and TP2, which are present in equimolar amounts, interact with dsDNA genome and form the nucleocapsid of the virus [13-16]. None of the TTV1 capsid proteins shows recognizable sequence similarity to structural proteins of other archaeal filamentous viruses [17] and virion organization of TTV1 also appears to be unique [18]. This prompted us to reevaluate the features of the structural proteins of TTV1 with the goal of understanding the provenance of this virus and its relationship to other viruses of archaea.

Analysis of the TP2, TP3 and TP4 sequences showed that despite the fact that over 30 years have passed since the TTV1 isolation these proteins remain to be ORFans without detectable homologs in other known viruses or cellular organisms. Although BLASTP searches [19] seeded 


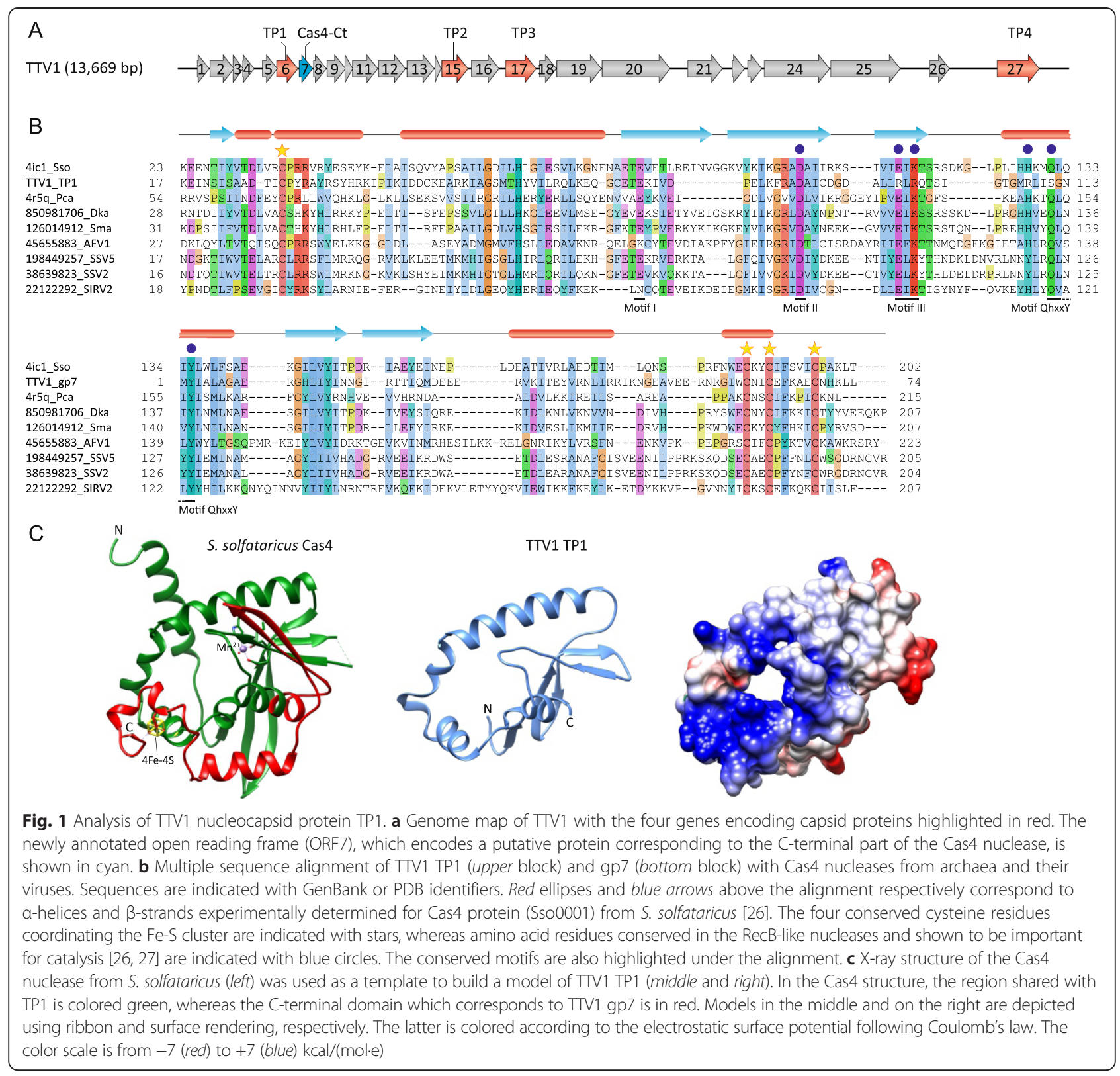

with the TP1 sequence also failed to identify homologs in public databases, more sensitive Hidden Markov modelbased HHpred analysis [20] resulted in a highly significant hit. Unexpectedly, TP1 was found to be homologous to Cas4 nuclease (PHA00619; $P=94.5, E=7.8 \mathrm{e}-02$ ), one of the proteins associated with the prokaryotic CRISPR-Cas immunity system $[21,22]$. The match encompassed $70 \%$ of the TP1 sequence (residues 11-90 out of 113) and corresponded to the $\mathrm{N}$-terminal half of the Cas4 proteins (Additional file 1: Figure S1A). Notably, the hit to nucleases of the PD-(D/E)XK superfamily was considerably weaker (PF12705; $P=70.9$ ). Multiple sequence alignment of TP1 with selected Cas4 proteins from cellular organisms and archaeal viruses has further extended the aligned region and confirmed the homology of these proteins (Fig. 1b). However, the C-terminal region that is conserved in Cas4 proteins remained unaccounted for. Additional analysis of the TTV1 genome showed that a region between nucleotides 1853 and 2074 (X14855), immediately downstream of the TP1 gene, encompasses a previously unannotated open reading frame (74 codons, denoted 7 in Fig. 1a) which corresponds to the missing $\mathrm{C}$-terminal part of the Cas4 proteins. HHpred analysis initiated with the gp7 sequence returned multiple significant hits to Cas4 proteins $(P=96.3, \mathrm{E}=4.9 \mathrm{e}-03$; Additional file 1: Figure $\mathrm{S} 1 \mathrm{~B})$ and multiple sequence alignment is fully consistent with this result (Fig. 1b). Collectively, genes encoding TP1 and gp7 reconstitute a full-length Cas4 gene and the split in 
the ancestral TTV1 Cas4 has apparently occurred within the QhxxY motif that is conserved in the Cas4 family and other RecB-like nucleases (Fig. 1b). The experimentally determined molecular weight of TP1 ( 14 kDa) [13] is consistent with the mass of this protein estimated from the sequence $(12.9 \mathrm{kDa})$. The molecular weight of the reconstituted, full-length Cas4 would be considerably larger $(21.6 \mathrm{kDa})$, confirming that the split between TP1- and gp7-encoding genes does not result from a sequencing error and that TP1 functions as a stand-alone protein. Although it is not known whether gp7 is expressed, this protein does not appear to be part of the TTV1 virion [13].

The Cas4 nuclease is widespread in Type I and Type II CRISPR-Cas systems and is believed to be involved in the acquisition of new spacers together with Cas1 and Cas2 proteins, and possibly additional CRISPR-associated functions [23-25]. Biochemical characterization of several Cas4 proteins has shown that they possess a broad spectrum of activities in vitro, including endonuclease, $5^{\prime} \rightarrow 3^{\prime}$ and $3^{\prime} \rightarrow 5^{\prime}$ exonuclease as well as ATPindependent DNA unwinding activities [26, 27]. Highresolution X-ray structures of two Cas4 proteins have been solved - one from Sulfolobus solfataricus [26] and the other from Pyrobaculum calidifontis [27]. The proteins consist of two domains the N-terminal RecB-like nuclease domain and the $\mathrm{C}$-terminal domain containing a Fe-S cluster coordinated by 4 conserved cysteine residues [26, 27] (Fig. 1c). Notably, the 4 conserved Cys residues are split between TP1 and gp7, with Cys1 located within TP1 and the 3 remaining Cys in gp7. Furthermore, not all active site residues characteristic of the RecB-like nucleases $[26,27]$ are preserved in TP1; in particular, the glutamate residue located in Motif III and involved in the coordination of a metal ion is replaced by an arginine in TP1 (Fig. 1b). Thus, TP1 lacks the Fe-S cluster and is unlikely to be catalytically active due to mutations in the active site, including the truncation of the Motif QhxxY.

To better assess the implications of the changes described above for the function of TP1, we built its structural model (Fig. 1c) based on the X-ray structure of Cas4 from S. solfataricus (Sso0001, PDB ID: 4ic1; [26]; the two proteins are $24 \%$ identical within the aligned regions). Analysis of the electrostatic charge distribution in TP1 model revealed a highly positively charged surface encompassing the $\mathrm{N}$-terminus of the protein, which might be important for DNA binding. Importantly, the corresponding surface in Cas4 is shielded by the Cterminal domain, suggesting that removal of this domain was a prerequisite for the transformation of the ancestral TTV1 Cas4 into a nucleocapsid protein.

\section{Concluding remarks}

Cas4 nuclease is a conserved component of the CRISPRCas systems. Beyond CRISPR-Cas, Cas4-like nucleases are also occasionally encoded in casposons, a recently discovered group of large transposable elements [28], as well as genomes of bacterial and archaeal viruses [29-31]. Among archaeal viruses, Cas4-like proteins are encoded by members of at least three different families, including Rudiviridae, Lipothrixviridae and Fuselloviridae (Fig. 1b), and in the case of rudivirus SIRV2, the protein has been shown to possess both $5^{\prime} \rightarrow 3^{\prime}$ exonuclease and endonuclease activities in vitro [29,32]. Thus, it appears likely that the ancestor of TTV1 encoded a functional Cas4 nuclease which could participate in certain aspects of genome replication or repair. At a certain point in evolution, the gene was truncated, possibly following the mutation(s) in the active site, and the region encoding the $\mathrm{N}$-terminal domain of the Cas4 nuclease evolved into a DNA-binding protein which was recruited as a new nucleocapsid protein of TTV1. Exaptation, a process whereby a function or a trait changes during evolution, is a major evolutionary phenomenon [33] that has also contributed to the evolution of viruses [34, 35]. Our finding that a Cas4-like nuclease has evolved into a viral CP provides another striking example of exaptation in virus evolution. More generally, it appears that viral CPs can occasionally evolve from functionally diverse proteins that originally had no involvement in formation of virus-like particles.

\section{Methods}

The non-redundant database of protein sequences at the NCBI was searched using the PSI-BLAST [19]. Protein sequences were aligned with Promals3D [36]. The alignment was visualized using Jalview [37]. Profile-against-profile searches were performed using HHpred [20] against different protein databases, including PFAM, PDB, CDD, and COG, which are available via the HHpred website. The hit to Cas4 profile (PHA00619; $P=94.5$ ) was obtained when the search was performed against the CDD database (the alignment of the hit is shown in the Additional file 1: Figure S1A). Structural modelling was done with Modeller v9.15 [38]. The resultant model (DOPE score of -14074.4) was then verified for stereochemical consistency using ProSA-web [39]; the Z-score was found to be -3.33 . The TP1 structural model and the template (PDB ID: 4ic1) structures were visualized using UCSF Chimera [40].

\section{Reviewers' reports}

Reviewer 1: Vivek Anantharaman (National Center for Biotechnology Information, National Library of Medicine, National Institutes of Health)

This is a straight forward paper describing that TP1 belongs to the Cas4 fold. I have a few questions and comments.

1) Line $72-74$ When the TP1 protein by itself is run through HHPRED it only hits a member of the REase (PDDEXK) fold (PF12705). Only when the C terminal 
gp7 sequence is added does the hit to Cas4 stand out. This needs to be clarified.

Authors' response: We performed HHpred searches against different databases accessible though the HHpred website, including PFAM, PDB (Protein Data Bank), CDD (Conserved Domain Database at NCBI), and COG (clusters of orthologous genes). This information has now been added to the Methods section. The hit to Cas4 profile (PHA00619; P =94.5) was specifically obtained when the search was performed against the CDD database (the alignment of the hit is shown in the Additional file 1: Figure $\mathrm{S1A})$. The hit to PDDEXK-like nucleases was considerably weaker $(P=70.9)$. This is now clarified in the revised manuscript.

2) Is there evidence that the gp7 protein (the $C$ terminal part) is not being made? If so please mention it. If not, the two proteins could function together as subunits and at least retain the metal binding role.

Authors' response: Unfortunately, the information regarding gp 7 expression is not available. However, even if the protein is expressed, it does not appear to be part of the TTV1 virion. We point this out in the revised manuscript.

3) Only the E among the conserved active site residues is changed to $R$. There are instances of REases that are active with changes to the E and Ref 25 shows QxxxY mutants retain their activity. The paper rightly says "unlikely" to be active in Line:105, but becomes more emphatic in attributing inactivity in the conclusion. Unless experimentally verified, one can only say it is most likely to be inactive.

Authors' response: We agree with the reviewer that caution should be exercised and removed the claim regarding the lack of nuclease activity from the Conclusions section.

Minor issues

3) The conserved $\mathrm{H}$ is also part of the active site (ref $25,26)$ and is not marked in the figure.

Authors' response: The conserved His is now also highlighted.

\section{Reviewer 2: Christine Orengo (Institute of Structural and Molecular Biology, University College London)}

The article presents an interesting analysis of the evolutionary relationship between a viral capsid protein and the Cas4 nuclease family. Capsid proteins have been found to derive from a range of different fold groups and this paper shows the recruitment of a protein with another type of structure, originally functioning as an enzyme, to a new function as a capsid protein. Sound methodology has been used but the authors should include more details on the significance of the matches they detect.

This is a brief but interesting paper demonstrating an evolutionary relationship between a viral capsid protein and a Cas4 nuclease. The TTV1 capsid protein studied has no significant sequence similarity to other archaeal virus proteins and the virion organisation is unique. The authors have detected this relationship using a wellestablished method, HHpred, which is probably the most powerful method available to date for identifying distant realtionships. Analysis of a multiple alignment generated using the sequences identified by the searches shows that the capsid protein has lost the catalytic residues necessary for the nuclease activity. A 3D model built from an available structure of Cas4 nuclease reveals a highly positively charged patch which may be implicated in DNA binding. This region is concealed by the Cterminal domain in Cas4 and the authors speculate that the removal of the $\mathrm{C}$ terminal region - detected by their studies - is necessary for the transformation of the Cas4 nuclease into a nucleocapsid protein. The manuscript summarises the literature on these proteins well and presents interesting observations but it would have been helpful to have more details of the results of the computational work. For example what E-value does HHpred give for the match ie how significant is the match.

Authors' response: The significance scores were provided in the Additional file 1: Figure S1. The HHpred probabilities of the TP1 and gp7 hits to Cas 4 were 94.53 (E-value = 0.078 ) and 96.31 (E-value $=0.0049)$, respectively. These values are now also indicated in the main text.

Similarly, what is the sequence similarity between the capsid protein and the Cas4 nuclease structure used to model it and what is the quality of the model built (eg DOPE score). This data should be given in the manuscript as it is necessary to judge the validity of the authors conclusions.

Authors' response: The sequence identity between TP1 and the template structure, the DOPE score as well as the ProSA-Web score, which was used to further verify the stereochemical consistency of the model, are now provided in the main text and the Methods section.

Minor issues

Details of the sequence search (eg E-value of the match) should be presented in the main text.

Authors' response: All this information is now provided.

\section{Reviewer 3: Mircea Podar (Biosciences Division, Oak Ridge National Laboratory)}

This is a very interesting finding about exaptation of Cas4 to serve as a structural viral protein. The article is well written and I have no criticism to the approach and data interpretation. This finding is important in the quest to understanding the evolution of viruses and how structural elements in capsids diversify across evolutionary distances.

It seems that only one strain of TTV1 is available, is that right? If so, are there perhaps TTV-type sequences 
present in metagenomic datasets? It would be valuable if such closely related viruses or viral genomes would be available as they would provide information on more recent changes/variants of the TP1, even finding variants in which the original enzymatic function of Cas4 may still be present. It is unclear if the HHPred search was also performed against metagenomic sequences.

Authors' response: Finding the intermediates with different variants of Cas4/TP1 genes would be indeed interesting. Unfortunately, no other TTV1-like viruses have been described thus far and, to the best of our knowledge, sequences closely matching TTV1 have never been reported in the metagenomics studies. Collection of all available metagenomes and their assembly into contigs is a considerable effort, which appears to be beyond the scope of the current short report.

\section{Additional file}

Additional file 1: Figure S1. Results of the HHpred search seeded with TP1 (A) and gp7 (B) sequences. H(h), a-helix; E(e), ß-strand; C(c), coil. (PDF $237 \mathrm{~kb})$

\begin{abstract}
Abbreviations
AFV1: Acidianus filamentous virus 1 (Lipothrixviridae); BLAST: basic local alignment search tool; CDD: conserved domain database; COG: clusters of orthologous genes; CP: capsid protein; CRISPR-Cas: Clustered Regularly Interspaced Short Palindromic Repeats/CRISPR-associated proteins; Dka: Desulfurococcus kamchatkensis; DOPE: discrete optimized protein energy; Pca: Pyrobaculum calidifontis; PDB: protein data bank; SIRV2: Sulfolobus islandicus rod-shaped virus 2 (Rudiviridae); Sma: Staphylothermus marinus F1; Sso: Sulfolobus solfataricus; SSV2 and SSV5: Sulfolobus spindle-shaped viruses 2 and 5 (Fuselloviridae); TTV1: Thermoproteus tenax virus 1.
\end{abstract}

\section{Competing interests}

The authors declare that they have no competing interests.

\section{Authors' contributions}

MK made the initial observation. MK, VC-K, DP and EVK participated in data analysis and writing of the manuscript. All authors read and approved the final version.

\section{Acknowledgements}

This work was supported by the Agence nationale de la recherche (ANR) program BLANC, project EXAVIR. EVK is supported by intramural funds of the US Department of Health and Human Services (to the National Library of Medicine).

\section{Author details}

${ }^{1}$ Department of Microbiology, Unité Biologie Moléculaire du Gène chez les Extrêmophiles, Institut Pasteur, Paris 75015, France. ${ }^{2}$ National Center for Biotechnology Information, National Library of Medicine, National Institutes of Health, Bethesda, MD 20894, USA.

Received: 4 September 2015 Accepted: 26 October 2015 Published online: 29 October 2015

\section{References}

1. Koonin EV, Senkevich TG, Dolja W. The ancient Virus World and evolution of cells. Biol Direct. 2006;1:29.

2. Krupovic M, Bamford DH. Order to the viral universe. J Virol. 2010;84(24):12476-9.

3. Raoult D, Forterre P. Redefining viruses: lessons from Mimivirus. Nat Rev Microbiol. 2008;6(4):315-9.
4. Forterre $P$, Krupovic M, Prangishvili D. Cellular domains and viral lineages. Trends Microbiol. 2014;22(10):554-8.

5. Jalasvuori M, Koonin EV. Classification of prokaryotic genetic replicators: between selfishness and altruism. Ann N Y Acad Sci. 2015:1341:96-105.

6. Krupovic M, Bamford DH, Koonin EV. Conservation of major and minor jellyroll capsid proteins in Polinton (Maverick) transposons suggests that they are bona fide viruses. Biol Direct. 2014;9(1):6.

7. Jalasvuori M, Mattila S, Hoikkala V. Chasing the origin of viruses: capsidforming genes as a life-saving preadaptation within a community of early replicators. PLoS One. 2015:10(5):e0126094.

8. Krupovic M, Bamford DH. Double-stranded DNA viruses: 20 families and only five different architectural principles for virion assembly. Curr Opin Virol. 2011;1(2):118-24.

9. Krupovic M, Quemin ER, Bamford DH, Forterre P, Prangishvili D. Unification of the globally distributed spindle-shaped viruses of the Archaea. J Virol. 2014;88(4):2354-8.

10. Abrescia NG, Bamford DH, Grimes JM, Stuart DI. Structure unifies the viral universe. Annu Rev Biochem. 2012;81:795-822.

11. DiMaio F, Yu X, Rensen E, Krupovic M, Prangishvili D, Egelman EH. Virology. A virus that infects a hyperthermophile encapsidates A-form DNA. Science. 2015;348(6237):914-7.

12. Koonin EV, Dolja W, Krupovic M. Origins and evolution of viruses of eukaryotes: the ultimate modularity. Virology. 2015;479-480:2-25.

13. Janekovic D, Wunderl S, Holz I, Zillig W, Gierl A, Neumann H. TTV1, TTV2 and TTV3, a family of viruses of the extremely thermophilic, anaerobic, sulfur reducing archaebacterium Thermoproteus tenax. Mol Gen Genet. 1983;192:39-45.

14. Reiter WD, Zillig W, Palm P. Archaebacterial viruses. Adv Virus Res. 1988:34:143-88.

15. Neumann H, Schwass V, Eckerskorn C, Zillig W. Identification and characterization of the genes encoding three structural proteins of the Thermoproteus tenax virus TTV1. Mol Gen Genet. 1989;217(1):105-10.

16. Neumann H, Zillig W. Coat protein TP4 of the virus TTV1: primary structure of the gene and the protein. Nucleic Acids Res. 1989;17(22):9475.

17. Prangishvili D, Krupovic M. A new proposed taxon for double-stranded DNA viruses, the order "Ligamenvirales". Arch Virol. 2012;157(4):791-5.

18. Prangishvili D. Archaeal viruses: living fossils of the ancient virosphere? Ann N Y Acad Sci. 2015;1341:35-40.

19. Altschul SF, Madden TL, Schaffer AA, Zhang J, Zhang Z, Miller W, et al. Gapped BLAST and PSI-BLAST: a new generation of protein database search programs. Nucleic Acids Res. 1997;25(17):3389-402.

20. Söding J. Protein homology detection by HMM-HMM comparison. Bioinformatics. 2005;21(7):951-60.

21. Makarova KS, Haft DH, Barrangou R, Brouns SJ, Charpentier E, Horvath P, et al. Evolution and classification of the CRISPR-Cas systems. Nat Rev Microbiol. 2011;9(6):467-77.

22. Makarova KS, Wolf YI, Koonin EV. The basic building blocks and evolution of CRISPR-CAS systems. Biochem Soc Trans. 2013;41(6):1392-400.

23. Koonin EV, Krupovic M. Evolution of adaptive immunity from transposable elements combined with innate immune systems. Nat Rev Genet. 2015;16(3):184-92.

24. Koonin EV, Makarova KS. CRISPR-Cas: evolution of an RNA-based adaptive immunity system in prokaryotes. RNA Biol. 2013;10(5):679-86.

25. Makarova KS, Aravind L, Wolf Yl, Koonin EV. Unification of Cas protein families and a simple scenario for the origin and evolution of CRISPR-Cas systems. Biol Direct. 2011;6:38.

26. Lemak S, Beloglazova N, Nocek B, Skarina T, Flick R, Brown G, et al. Toroidal structure and DNA cleavage by the CRISPR-associated [4Fe-4S] cluster containing Cas4 nuclease SSO0001 from Sulfolobus solfataricus. J Am Chem Soc. 2013;135(46):17476-87.

27. Lemak S, Nocek B, Beloglazova N, Skarina T, Flick R, Brown G, et al. The CRISPR-associated Cas4 protein Pcal_0546 from Pyrobaculum calidifontis contains a [2Fe-2S] cluster: crystal structure and nuclease activity. Nucleic Acids Res. 2014;42(17):11144-55.

28. Krupovic M, Makarova KS, Forterre P, Prangishvili D, Koonin EV. Casposons: a new superfamily of self-synthesizing DNA transposons at the origin of prokaryotic CRISPR-Cas immunity. BMC Biol. 2014;12:36.

29. Guo $Y$, Kragelund BB, White MF, Peng X. Functional characterization of a conserved archaeal viral operon revealing single-stranded DNA binding, annealing and nuclease activities. J Mol Biol. 2015;427(12):2179-91. 
30. Hooton SP, Connerton IF. Campylobacter jejuni acquire new host-derived CRISPR spacers when in association with bacteriophages harboring a CRISPR-like Cas4 protein. Front Microbiol. 2014;5:744.

31. Prangishvili D, Koonin EV, Krupovic M. Genomics and biology of Rudiviruses, a model for the study of virus-host interactions in Archaea. Biochem Soc Trans. 2013;41(1):443-50.

32. Gardner AF, Prangishvili D, Jack WE. Characterization of Sulfolobus islandicus rod-shaped virus 2 gp19, a single-strand specific endonuclease. Extremophiles. 2011;15(5):619-24.

33. Gould SJ, Vrba ES. Exaptation - a missing term in the science of form. Paleobiology. 1982;8(1):4-15.

34. Kazlauskas D, Venclovas C. Herpesviral helicase-primase subunit UL8 is inactivated B-family polymerase. Bioinformatics. 2014;30(15):2093-7.

35. Yutin N, Faure G, Koonin EV, Mushegian AR. Chordopoxvirus protein F12 implicated in enveloped virion morphogenesis is an inactivated DNA polymerase. Biol Direct. 2014;9(1):22

36. Pei J, Grishin NV. PROMALS3D: multiple protein sequence alignment enhanced with evolutionary and three-dimensional structural information. Methods Mol Biol. 2014;1079:263-71.

37. Waterhouse AM, Procter JB, Martin DM, Clamp M, Barton GJ. Jalview Version 2-a multiple sequence alignment editor and analysis workbench. Bioinformatics. 2009;25(9):1189-91.

38. Marti-Renom MA, Stuart AC, Fiser A, Sanchez R, Melo F, Sali A. Comparative protein structure modeling of genes and genomes. Annu Rev Biophys Biomol Struct. 2000;29:291-325.

39. Wiederstein M, Sippl MJ. ProSA-web: interactive web service for the recognition of errors in three-dimensional structures of proteins. Nucleic Acids Res. 2007:35(Web Server issue):W407-410.

40. Pettersen EF, Goddard TD, Huang CC, Couch GS, Greenblatt DM, Meng EC, et al. UCSF Chimera-a visualization system for exploratory research and analysis. J Comput Chem. 2004;25(13):1605-12.

\section{Submit your next manuscript to BioMed Central and take full advantage of:}

- Convenient online submission

- Thorough peer review

- No space constraints or color figure charges

- Immediate publication on acceptance

- Inclusion in PubMed, CAS, Scopus and Google Scholar

- Research which is freely available for redistribution 